\title{
Development of a fully injectable calcium phosphate cement for orthopedic and dental applications
}

\author{
MANOJ KOMATH* and H K VARMA \\ Biomedical Technology Wing, Sree Chitra Tirunal Institute for Medical Sciences and Technology, Thiruvanantha- \\ puram 695 012, India
}

MS received 27 January 2003; revised 21 April 2003

\begin{abstract}
A study on the development of a fully injectable calcium phosphate cement for orthopedic and dental applications is presented. The paper describes its characteristic properties including results of biocompatibility studies.

A conventional two-component calcium phosphate cement formulation (having a powder part containing dry mixture of acidic and basic calcium phosphate particles and a liquid part containing phosphate solution) is modified with a biocompatible gelling agent, to induce flow properties and cohesion. The quantity of the gelling agent is optimized to get a viscous paste, which is smoothly injectable through an 18-gauge needle, with clinically relevant setting parameters.

The new formulation has a setting time of $20 \mathrm{~min}$ and a compressive strength of $11 \mathrm{MPa}$. The X-ray diffraction, Fourier transform infrared spectrometry, and energy dispersive electron microprobe analyses showed the phase to be hydroxyapatite, the basic bone mineral. Scanning electron microscopy revealed a porous structure with particle sizes of a few micrometers. The cement did not show any appreciable dimensional or thermal change during setting. The injectability is estimated by extruding through needle and the cohesive property is assessed by water contact method. The cement passed the in vitro biocompatibility screening (cytotoxicity and haemolysis) tests.
\end{abstract}

Keywords. Calcium phosphate cements; hydroxyapatite; bioceramics; bone substitute; orthopedic; dental.

\section{Introduction}

Calcium phosphate cements (CPCs) are the emerging class of bone substitute materials. They are identified as excellent alloplastic material for osseous augmentation because of the unique combination of osteoconductivity, biocompatibility and mouldability. Calcium phosphate cements are more attractive than hydroxyapatite ceramic granules because they can be molded and shaped to fill intricate bony cavities or narrow dental defect sites. The non-toxic and non-carcinogenic properties of CPCs make them more advisable than PMMA bone cements. Other interesting properties are non-exothermic setting and negligible shrinkage. In addition, they show excellent in vivo resorption.

The principle of CPCs is the cementing action of acidic and basic calcium phosphate compounds on wetting with an aqueous medium. The cement formulation essentially consists of two parts - a powder mix containing dry calcium phosphate particles and a wetting medium prepared with de-ionized distilled water. Mixing of these in suitable proportion gives a self-setting mass. The setting time can be adjusted by adding manipulator compounds

*Author for correspondence (physiologically acceptable phosphates) to the wetting medium. The chemical phase of the set mass depends on the net $\mathrm{Ca}-\mathrm{P}$ ratio in the ingredients and when this is adjusted to $1 \cdot 67$, hydroxyapatite (the basic bone mineral with the chemical formula $\left.\mathrm{Ca}_{10}\left(\mathrm{PO}_{4}\right)_{6}(\mathrm{OH})_{2}\right)$ will result.

Several CPC compositions based on various calcium and phosphate compounds were formulated and their characteristics and in vitro and in vivo properties had been investigated during the last decade (Driessens et al 1995, 1998; Bohner 2000). In recent years, a large number of animal and clinical studies have been conducted to prove the success of CPCs in various surgical procedures (Reddi et al 1999; Lew et al 2000; Turk and Parhiscar 2000; Bohner 2001; Cherng et al 2001; Verheggen and Merten 2001; Baker et al 2002; Ooms et al 2002; Sugawara et al 2002). Larsson and Bauer (2002) gives an extensive review of the use of calcium phosphate cements in fracture fixation.

Most of the surgical demonstrations were done using $\mathrm{CPC}$ in putty form, by applying the mix at directly accessible bony defects. However, requirements of delivering the material through needles/applicators often arise in actual clinical practice, particularly in the cases of narrow defects and sites of limited accessibility. Procedures like in situ fracture fixation in orthopaedics, filling root canals and sealing furcation perforation in endodontics 
and vertebroplasty in spinal surgery are examples. CPCs in putty form inherently lack viscous flow and cohesive property and hence it is difficult to inject them through narrow needles using a syringe or applicator.

The lack of viscous flow is a serious limitation for CPCs, as far as injectability is concerned. The cement mix can be made as loose paste by increasing the wetting ratio, but it will be of limited help. The liquidous part will get pushed-out through the needle and major portion of the particulate mass remain inside the syringe. Moreover, high wetting ratio increases the setting time to unacceptable values. The absence of cohesive property leads to 'washing-out' of the unset cement if exposed to an aqueous environment. The setting properties are affected when blood or body fluids are present at the site.

Despite these problems, some researchers tried injecting CPCs in paste form. The most notable among such reports is that of Constantz et al (1995). They achieved a remarkable success in the minimally invasive management of acute fracture of the radius through percutaneous administration of CPC. Recent studies show that injection of CPC is adopted for treating calcaneal bone cyst and pathological fracture cases (Csizy et al 2001), for tibial plateau fracture (Lobenhoffer et al 2002), in balloon vertebroplasty for direct restoration of traumatic thoracolumbar vertebral fractures (Verlaan et al 2002) and in filling the bone defects around oral implants (Comuzzi et al 2002). It is to be noted that in all these attempts loose pastes of CPC is used for injection. A fully injectable formulation (i.e. self-setting CPC mix having viscous flow so as to extrude out fully from a syringe through an injection needle) is still a challenge in the design of CPCs.

The authors have succeeded in developing a fully injectable calcium phosphate cement formulation, by incorporating gelling agents (Komath and Varma 2002). The work is done as a part of the product development activity of the Sree Chitra Tirunal Institute for Medical Sciences and Technology. This paper describes the development, material properties and biological screening of the new cement formulation.

\section{Experimental}

\subsection{Cement formulation}

The new formulation is based on the apatitic calcium phosphate cement composition already reported (Komath et al 2000). The cement powder contained tetracalcium phosphate (TTCP) and dicalcium phosphate dihydrate (DCPD) particles of size in the range of $100 \mu$, mixed in equimolar ratio. The wetting medium used was distilled water with $\mathrm{Na}_{2} \mathrm{HPO}_{4}$ as the setting accelerator in an optimized concentration of $0.2 \mathrm{M}$. The basic composition was not altered but for the addition of the gelling agent.
Some workers had suggested the use of gelling agents for improving the cohesion and handling properties of calcium phosphate cement putty (Ishikawa et al 1997; Khairoun et al 1999). The ability of the gelling agents to impart viscous flow on wetting is utilized in the present work to convert the CPC putty into fully injectable consistency. Many gelling agents are known but two stringent requirements arise in this case-the additive should be biocompatible and it should not affect the setting properties of the cement.

A few candidate compounds, with limited to good biocompatibility status, were tried for the purpose-mainly, glycerin, derivatives of cellulose and salts of alginic acid. On adding glycerin and cellulose derivatives to the formulation, sticky pastes resulted, which did not set properly. Phase analyses done indicated poor conversion of the ingredients to hydroxyapatite. The complete phase conversion is the prime requirement for calcium phosphate cements (Driessens et al 1995, 1998), which also ensures the biocompatibility. It was observed that the salts of alginic acid do not affect the cement setting drastically, though the rate of phase conversion gets delayed to certain extent depending on the ratio of the compound in the formulation. Thus, the salts of alginic acids were found better than other candidate gelling agents. They have proven toxicological safety (Sutherland 1991) and suggested pharmacologically beneficial effect on bone replacement (Ishikawa et al 1997) as added advantages.

The optimum quantity of the gelling agent required in the formulation was found by adding it in different weight ratios (up to $5 \% \mathrm{w} / \mathrm{w}$ in dry powder form) to the powder part of the cement and monitoring the change in properties. The volume of the wetting liquid required to make the paste increased with higher concentrations of the gelling agent (the conventional formulation was optimized for $0.5 \mathrm{ml}$ wetting medium per gram of powder). Increasing the quantity of the gelling agent imparted excellent flow properties to the cement, but prolonged the setting time. As the aim of the modification is to induce injectability, the criterion for cement consistency was set as the smooth flow through 18 -gauge needle $(0.85 \mathrm{~mm}$ inner diameter) when used with a $3 \mathrm{ml}$ syringe. The gelling agent ratios of $2 \% \mathrm{w} / \mathrm{w}$ and above met this requirement. However, the consequent prolongation in cement setting is of concern because setting times above $20 \mathrm{~min}$ are clinically not acceptable. The gelling agent ratios $1 \% \mathrm{w} / \mathrm{w}$, $2 \% \mathrm{w} / \mathrm{w}$ and $3 \% \mathrm{w} / \mathrm{w}$ gave the setting times as $12 \mathrm{~min}$, $20 \mathrm{~min}$ and $50 \mathrm{~min}$, respectively (the conventional cement sets at $10 \mathrm{~min}$ ). Compromising the flow properties and setting time, the optimum ratio of the gelling agent was decided to be $2 \% \mathrm{w} / \mathrm{w}$ of the powder part.

The optimized cement formulation was prepared by initially mixing $100 \mu$ particles of tetracalcium phosphate (TTCP) and dicalcium phosphate dihydrate (DCPD) in equimolar ratio and thereafter adding the gelling agent to it in dry powder form in a ratio of $2 \% \mathrm{w} / \mathrm{w}$. This 
was sterilized by gamma irradiation and preserved in a desiccator. The liquid part was prepared as $0.2 \mathrm{M}$ solution of $\mathrm{Na}_{2} \mathrm{HPO}_{4}$ in deionized distilled water and steamsterilized before use.

\subsection{Characterization of the cement}

The cement paste was prepared in a watch glass by taking a known weight of the powder and adding the liquid drop-wise till the powder got fully wet. The optimum wetting ratio was found to be $0.8 \mathrm{ml} / \mathrm{g}$. The wet mass was kneaded with a steel spatula to get a smooth paste. This was subjected to various tests and analyses.

The setting time of the cement was assessed using a custom fabricated Vicat type apparatus (Philips 1991; Komath et al 2000). It consisted of a steel needle (diameter, $1 \mathrm{~mm}$ ) moving along a vertical axis under a constant load of $100 \mathrm{gf}$, and a small plastic tray of $5 \mathrm{~mm}$ depth to hold the paste. The distance traveled by the tip can be measured to $0.01 \mathrm{~mm}$ accuracy using an attached dial gauge. The cement was mixed and filled in the tray and the needle was allowed to penetrate into it. The length of travel of the needle tip was recorded. Measurements were repeated at time intervals of $30 \mathrm{~s}$. The time period at which the needle ceases to penetrate the cement mass denotes the setting time.

Compressive strength of the set cement was measured in an Instron (model 1193) Universal Testing Machine as per ASTM Standard F 451-95. The cement samples were molded in the form of cylindrical pellets of $6 \mathrm{~mm}$ diameter and $12 \mathrm{~mm}$ height by injecting into steel moulds. After setting, they were incubated in $100 \%$ humidity for $24 \mathrm{~h}$ and dried before analysis. The pellets were compressed along their height in between the platens of the machine at a cross-head speed of $1 \mathrm{~mm} / \mathrm{min}$. The compressive strength was calculated from the break load and the dimensions of the pellets.

The phase analysis of the set cement was carried out using Siemens D 5005 X-ray diffractometer. The set cement mass was kept in $100 \%$ humidity at physiological conditions for $24 \mathrm{~h}$ and then dried and powdered for analysis. The powder is also subjected to FTIR spectrometry (Impact 410 , Nicolet) by $\mathrm{KBr}$ pellet technique.

The microstructure of the cement was observed in scanning electron microscope (Hitachi SEM model S2400), after breaking the set mass across and coating it with gold. An energy dispersive electron microprobe system (Oxford EDS system) attached to the SEM is used to determine the elemental composition. This was done on samples coated with carbon and all possible elements were searched. The $\mathrm{Ca} / \mathrm{P}$ ratio was determined quantitatively, where Wollastonite and $\mathrm{GaP}$ standards were used for $\mathrm{Ca}$ and $\mathrm{P}$, respectively.

The setting temperature was monitored in a differential thermal analyser (SDT 2960, TA Instruments) in isother- mal mode with alumina as the standard. The change in dimensions on setting was assessed by molding the cement in regular geometry and known dimensions, and measuring the set shape.

\subsection{Evaluation of injectability and cohesive property}

The viscous and cohesive properties are of major interest in the case of an injectable cement. As there are no standard procedures that exist to assess these properties, indigenous test methods were developed for the purpose.

2.3a Injectability: A modified version of the method adopted by Khairoun et al (2002) is employed to assess the injectability quantitatively. The evaluation is done by measuring the load required to extrude the unset cement through a 18-gauge needle. The conventional cement (without gelling agent) and the new injectable cement were compared in this way.

A $3 \mathrm{ml}$ syringe (of inner diameter $8.5 \mathrm{~mm}$ and plunger travel length of $5 \mathrm{~cm}$ ) was taken and fitted with a needle of size 18-gauge (0.85 mm ID and $1.45 \mathrm{~mm} \mathrm{OD).} 2 \mathrm{cc}$ of the mixed cement was filled in the syringe and tightly packed by pressing the plunger. The volume of the cement was sufficient to fill a length of $20 \mathrm{~mm}$ inside the syringe. The syringe was fitted vertically in a fixture and put under the platen of the Universal Testing Machine set in compressive mode. The compressive force was recorded during the extrusion of the cement at a cross-head speed of $1 \mathrm{~mm} / \mathrm{min}$ and plotted against the plunger travel length.

2.3b Cohesive property: It was observed that the cohesive property of the cement improved considerably on adding the gelling agent. A qualitative estimation was done following the method suggested by Ishikawa et al (1997). The cohesion of conventional cement and the new injectable cement were compared, by exposing them to water in unset condition.

The cement samples were cast as $6 \mathrm{~mm}$ pellets and transferred immediately to a petri dish kept under an optical microscope. After $4 \mathrm{~min}$, distilled water taken in a pipette was dispensed over the pellet continuously and slowly till the pellet got immersed. The condition of the pellet was observed in the microscope. The addition was continued and the pellet was visually monitored. The extent of dispersing or crumbling gave an idea about the cohesive nature of the cement mass.

\subsection{Biocompatibility screening}

The optimized cement was subjected to standard cytotoxicity and haemolysis screening tests to assess the biocompatibility. 
The cell culture cytotoxicity studies were done in direct contact method (ISO 10993-5, 1999) using mouse fibroblast cells. The test samples were prepared by setting the cements in the form of discs of $10 \mathrm{~mm}$ diameter and $2 \mathrm{~mm}$ thickness. They were cleaned ultrasonically and sterilized by autoclaving.

The culture medium consisted of Eagle's MEM (containing glutamine) and fetal bovine serum (10\%) along with antibiotics. Mouse fibroblast cells (L929, supplied by NCCS, Pune) were subcultured and seeded into 24 multiwell dishes to form a monolayer. The samples were placed gently onto the cells and incubated at $37^{\circ} \mathrm{C}$ under $5 \% \mathrm{CO}_{2}$ for $24 \mathrm{~h}$. The morphology of the cells in the vicinity of the material was examined under a phase contrast microscope (Leica, Germany). Test was done in duplicate, along with control samples.

The haemolytic potential of the cement was assessed, by exposing blood to the extract of the material in saline and assessing the lysis of red blood cells spectrophotometrically (as per ANSI/ADA Document No. 41, 1982). The samples for these tests were set in the form of pellets and preserved at $100 \%$ humidity for $24 \mathrm{~h}$ at physiological conditions. Sterilization was done by autoclaving at $120^{\circ} \mathrm{C}$ for $20 \mathrm{~min}$.

The extract of $3 \mathrm{~g}$ sample was prepared in $6 \mathrm{ml}$ normal saline and incubated at $37^{\circ} \mathrm{C}$ for $30 \mathrm{~min} .0 \cdot 12 \mathrm{ml}$ of diluted rabbit blood (fresh rabbit blood collected in $2 \%$ potassium oxalate solution and diluted at $1: 1$ with normal saline) was added to the extract. Negative control used was $0.2 \mathrm{ml}$ diluted blood in $10 \mathrm{ml}$ normal saline alone, and the positive control was $0.2 \mathrm{ml}$ diluted blood in $10 \mathrm{ml}$ of $0.1 \%$ sodium carbonate solution. The solutions were incubated at $37^{\circ} \mathrm{C}$ for $1 \mathrm{~h}$ and then centrifuged at $500 \mathrm{~g}$ (1800 rpm). The spectroscopic absorbance at $545 \mathrm{~nm}$ of the supernatant was measured in a spectrophotometer. The test was done in triplicate to take the average value and to calculate the percentage haemolysis.

$$
\% \text { Haemolysis }=\frac{\text { Mean of test OD }- \text { OD of negative control }}{\text { OD of positive control }- \text { OD of negative control }} \times 100 \text {. }
$$

\section{Results and discussion}

\subsection{Characteristics of the cement}

It was found that the cement gets set at about $20 \mathrm{~min}$ after mixing. The mean compressive strength was measured to be $11.12 \mathrm{MPa}$, a value which compares with that of trabecular bone (table 1). Both the setting time and compressive strength suit the clinical requirement (Driessens et al 1995).

The phase composition of the cement can be ascertained from X-ray diffraction. The XRD spectrum of the cement is shown in figure 1 , along with the spectrum of bone mineral (deproteinated bovine bone) and the standard lines of hydroxyapatite (JCPDS Data: 9-432). It is evident that the ingredients of the cement get converted to hydroxyapatite. The close similarity of the spectrum of the cement to that of the bone mineral is notable.

The FTIR analysis (figure 2) showed all typical absorption characteristics of hydroxyapatite (Rehman and Bonfield 1995). In addition, some carbonate content also was seen, which is an indication of the presence of carbonate apatite. This might have originated through the absorption of carbon dioxide from the atmosphere.

The elemental analysis through electron microprobe (EDS system) showed the presence of only oxygen, calcium and phosphorous. The $\mathrm{Ca} / \mathrm{P}$ atomic ratio found is 1.80 , which is higher than that of the stoichiometric hydroxyapatite (1.67). This apparent excess of calcium might have resulted by the substitution of carbonate ions in the phosphate moieties.

The surface micromorphology can be seen in scanning electron micrograph (figure 3). The precipitated cement particles were of the order of a few microns and the mass was found porous. The bulk density was measured to be $1.22 \mathrm{~g} / \mathrm{cc}$, whereas that of dense hydroxyapatite, $3 \cdot 16 \mathrm{~g} / \mathrm{cc}$.

The phase composition indicates the clinical advantage of the cement. The hydroxyapatite phase has proven osteoconductive properties and the microstructure of the cement is favourable for in vitro material resorption and bone ingrowth (Driessens et al 1995).

No shrinkage was observed (within a measurement accuracy of $\pm 0.05 \mathrm{~mm}$ ) in the cement mass set inside the dies of regular geometry and known dimensions and preserved in $100 \%$ humidity. The set cement, on drying up, showed an average linear shrinkage of less than $0.5 \%$.

Observations on the setting temperature of the cement in DTA (figure 4) with respect to the reference sample of same weight at isothermal conditions did not show any

Table 1. Results of the compressive strength studies on the set cement.

\begin{tabular}{lcc}
\hline Trial no. & $\begin{array}{c}\text { Break } \\
\text { load }(\mathrm{N})\end{array}$ & $\begin{array}{c}\text { Compressive } \\
\text { strength }(\mathrm{MPa})\end{array}$ \\
\hline 1 & 262 & $9 \cdot 283$ \\
2 & 300 & $10 \cdot 605$ \\
3 & 300 & $10 \cdot 605$ \\
4 & 375 & $13 \cdot 248$ \\
5 & 393 & $13 \cdot 909$ \\
6 & 356 & $12 \cdot 587$ \\
7 & 279 & $9 \cdot 878$ \\
8 & 317 & $11 \cdot 200$ \\
9 & 298 & $10 \cdot 539$ \\
10 & 298 & $10 \cdot 539$ \\
11 & 317 & $11 \cdot 200$ \\
12 & 279 & $9 \cdot 878$ \\
Mean value & & $11 \cdot 120$ \\
S.D. & & $1 \cdot 36$ \\
\hline
\end{tabular}


exothermic reaction. Instead, a decrease of $\sim 1{ }^{\circ} \mathrm{C}$ could be seen.

The lack of exothermicity and shrinkage is a remarkable advantage over injectable acrylic bone cements, which are used currently for prosthesis fixation and vertebroplasty. The acrylic based formulations ail from strong exothermic setting reaction (which elevates the tempera- ture to $\sim 100^{\circ} \mathrm{C}$ ) and shrinkage causing eventual detachment from the apposing bony region (Driessens et al 1995).

\subsection{Consistency and flow properties}

The result of the injectability study is given in figure 5 . In the case of the conventional cement, the force required

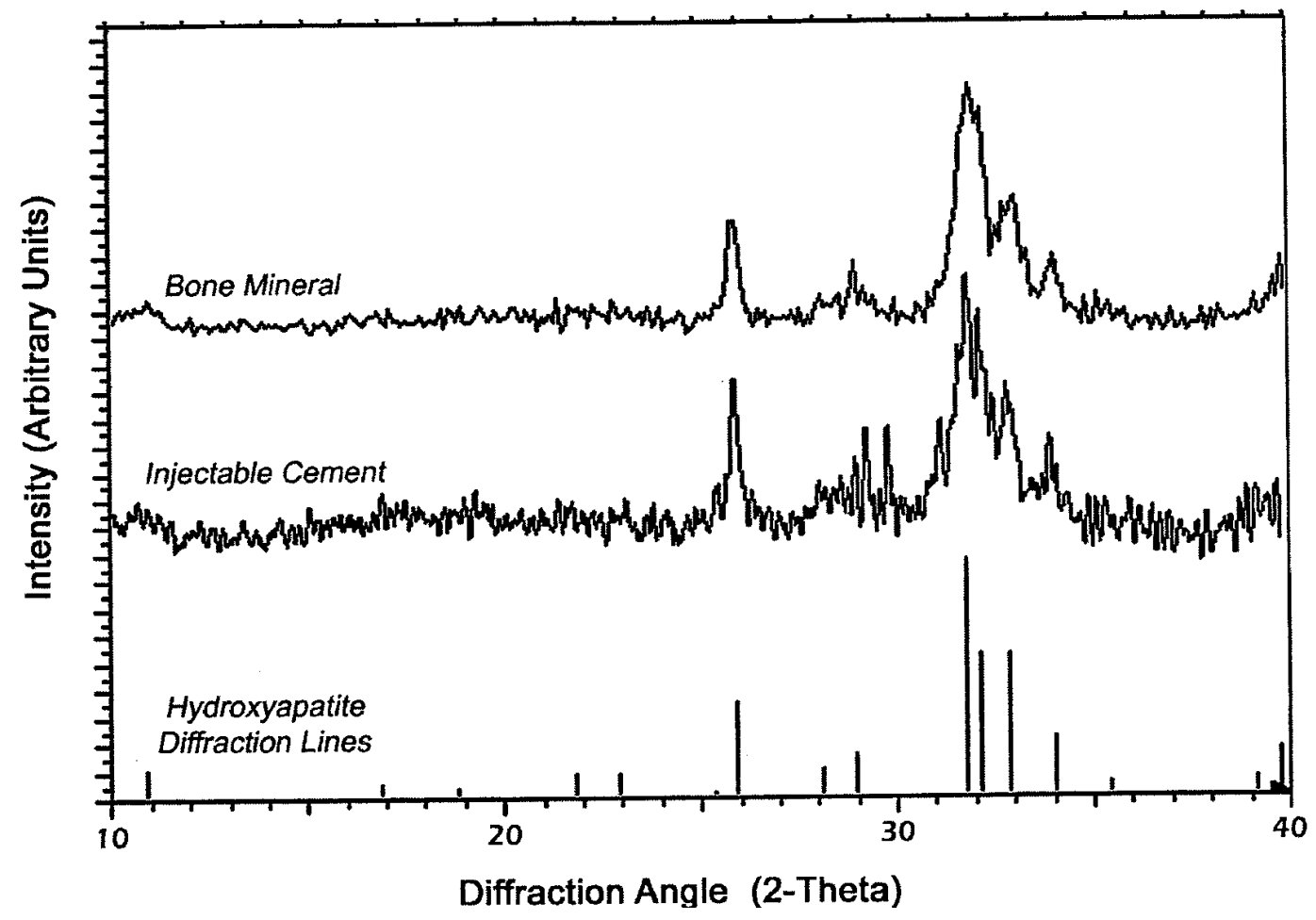

Figure 1. The X-ray diffraction spectrum of the new injectable cement along with the spectrum of bone mineral and standard diffraction lines of hydroxyapatite.

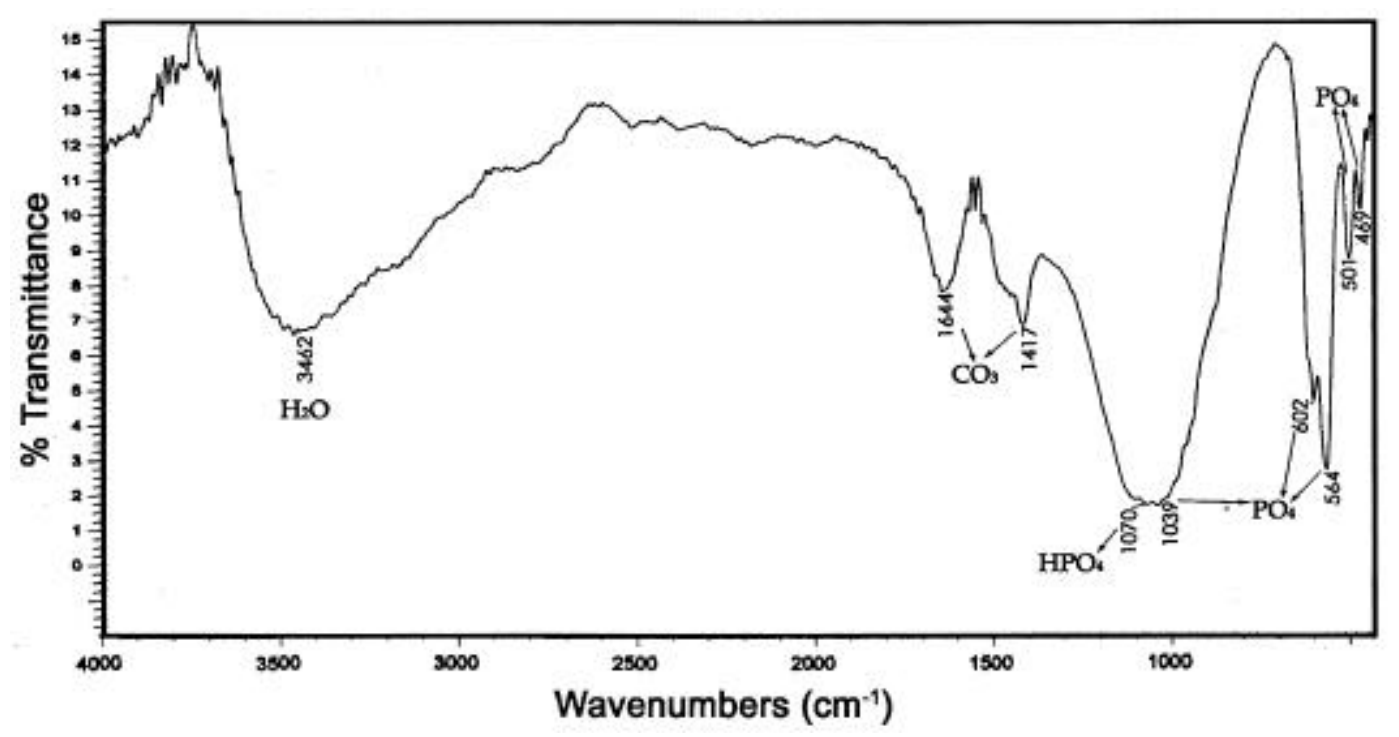

Figure 2. The FTIR spectrum of the new injectable cement. 
to extrude the mass exceeds the maximum thumb force after a few millimeters of plunger travel. It is clear that the cement mass cannot be fully injected manually. Meanwhile, the new injectable cement could

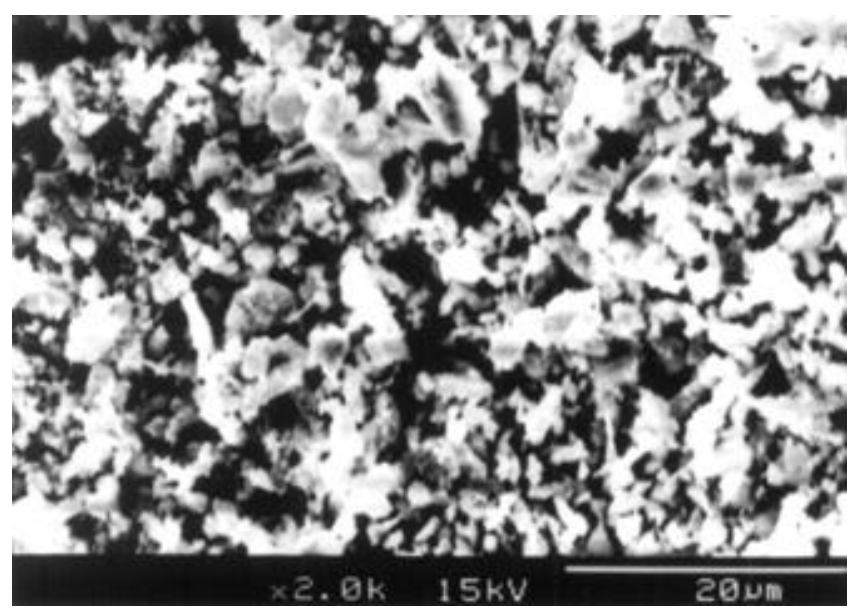

Figure 3. Scanning electron micrograph of the cement surface.

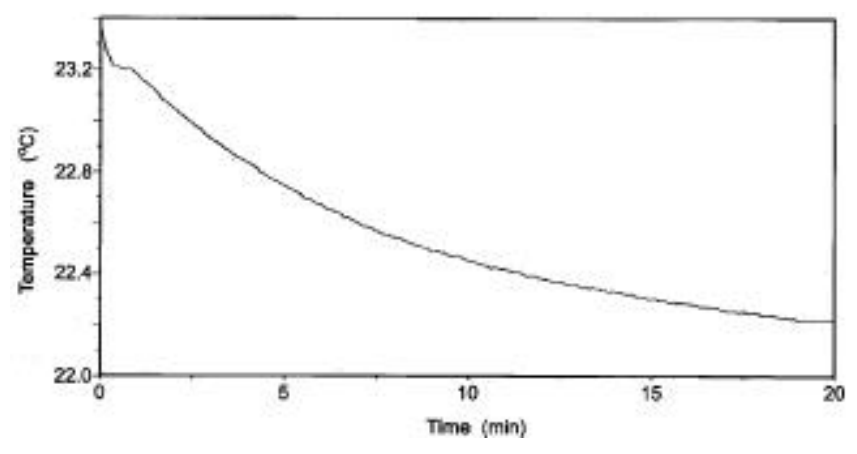

Figure 4. The DTA graph of the setting cement.

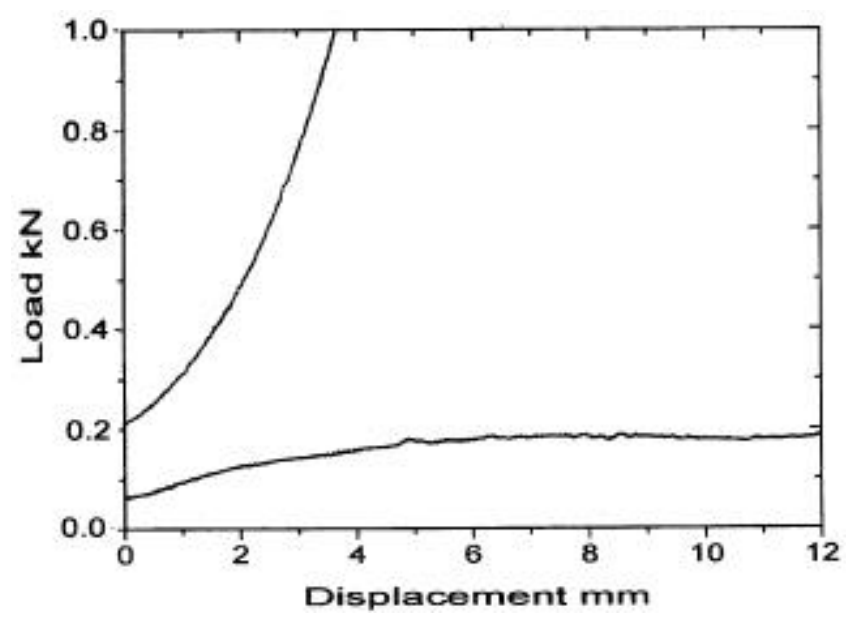

Figure 5. The displacement-load graph of the unset cement extrusion through an 18 -gauge needle from a $3 \mathrm{ml}$ syringe. The maximum average force that can be exerted by the thumb on the syringe is marked. be extruded throughout the length of the filling under constant load, which is well below the maximum thumb force.

Photographs of the unset cement cast in water, compiled as figure 6 , demonstrate the cohesive property of the new injectable cement. The conventional CPC begins to crumble on adding water and disperses in due course. It is observed that the setting of the mixed mass gets impaired when immersed in water. The new injectable cement shows good cohesion and retains its shape when immersed in water, except for slight bulging at the periphery. The mass is observed to set inside water, though with a prolonged setting time. The cohesive property is highly significant in clinical application that it can protect the unset cement mass from getting washed away at wet (or bleeding) surgical sites.

\subsection{Biocompatibility}

The biocompatibility screening showed highly promising results. The haemolytic potential is found to be $0.78 \%$ whereas the limit to pass the test is $5 \%$. The image taken during the direct contact cytotoxicity test is shown (figure 7). The mouse fibroblast cells around the material remained in the same normal morphology like the cells cultured without any sample, showing the non-toxic nature of the test specimens.

The clearance in haemolysis and cytotoxicity tests qualifies the new injectable cement for pre-clinical trials.

\section{Conclusions}

The present work demonstrates the possibility of formulating a fully injectable calcium phosphate cement. The desired properties of injectability and cohesion were achieved by incorporating a gelling agent. The addition of the gelling agent does not alter the characteristics of the cement, but for a prolongation in setting time. The cement neither possesses setting exotherm nor it shows any significant shrinkage.

The XRD and FTIR analyses showed that cement mass get converted into hydroxyapatite, which is closely similar to the mineral phase of vertebrate bone. The set cement has sufficient mechanical strength, comparable to that of trabecular bone. These features make the material suitable for clinical applications as bone filler.

Tests on the consistency established that the mixed cement could be passed fully through an 18-gauge needle from a $3 \mathrm{ml}$ syringe. A considerable improvement in cohesive property could be observed on adding the gelling agent. The mixed cement was seen to keep the shape when immersed in water and undergo setting eventually.

The new injectable cement passed the biocompatibility screening tests. The detailed toxicological and efficacy tests of this product are in progress. 


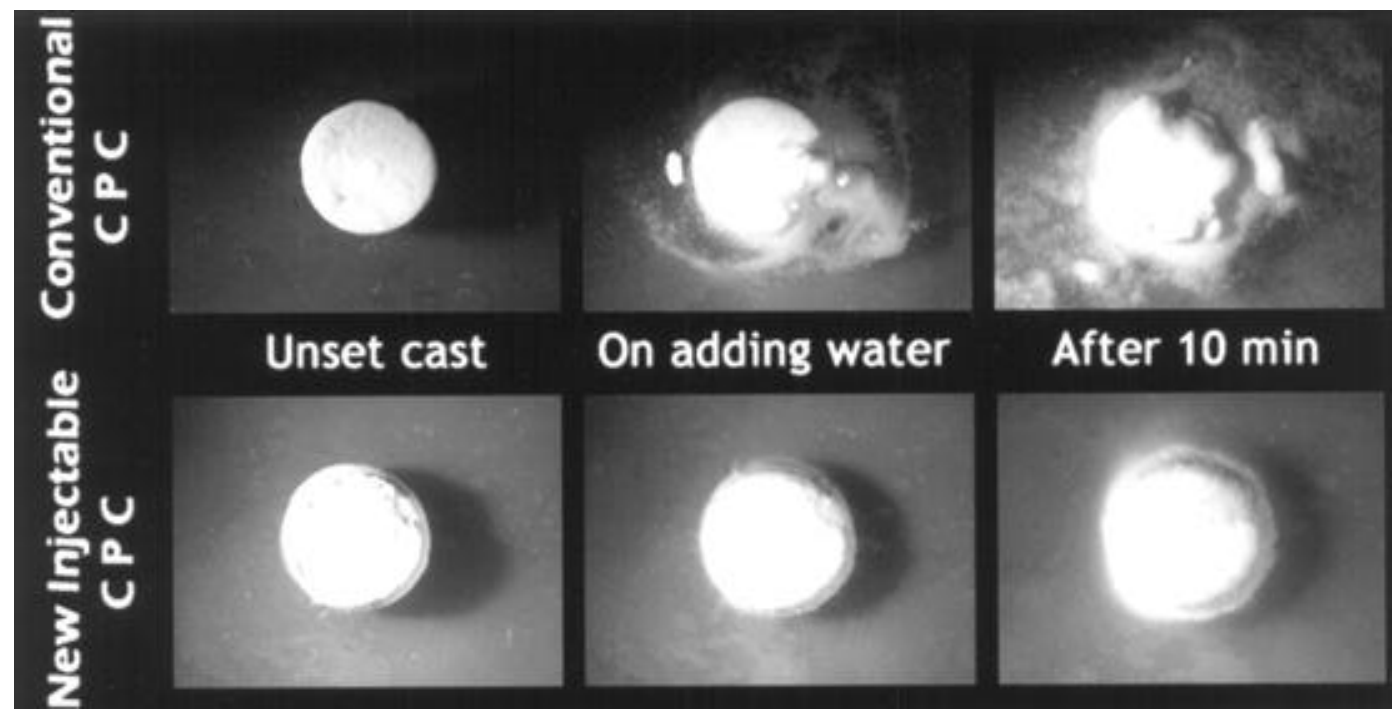

Figure 6. A comparison of the cohesive properties of the conventional cement and the new injectable cement. The behaviour of the unset cement pellets inside water is shown in sequence.

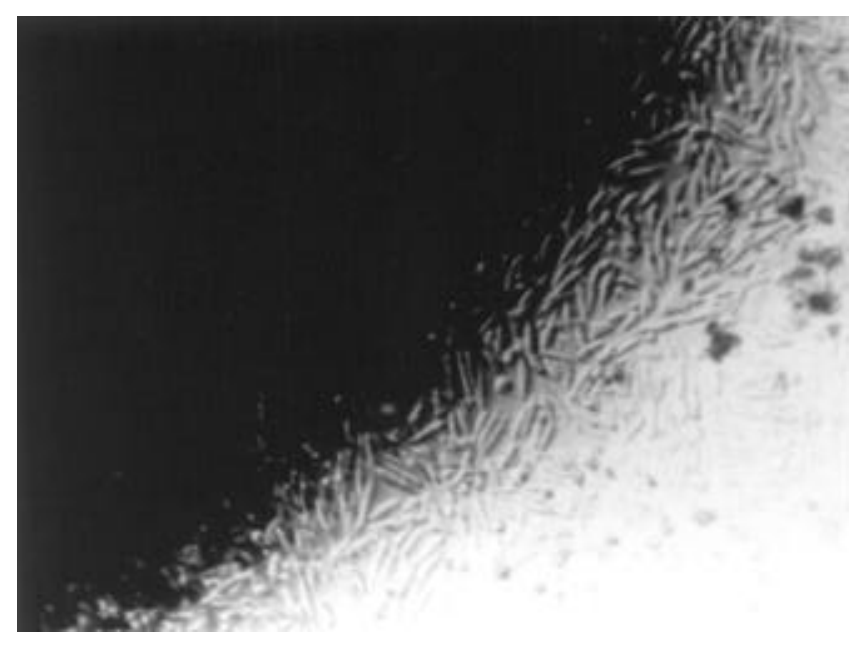

Figure 7. Mouse fibroblast (L929) cells in direct contact with the cement.

This product provides medical professionals with a self setting, osteoconductive paste for various skeletal repair applications, which can be injected to the defect site. The viscous properties of the cement is such that disposable syringes can be used for delivery, eliminating the need for special applicators. The mixed cement has sufficient cohesive property that body fluids will not disturb the setting. The fully injectable calcium phosphate cement will be a candidate material for procedures like in situ fracture fixation in orthopedics and vertebroplasty in spinal surgery, filling root canals and sealing endodontic perforations in dentistry.

\section{Acknowledgements}

Thanks are due to Dr T V Kumari, Mr Jacob Beboy, Mr P R Hari and Mrs C Radhakumari for conducting the various tests and analyses. The authors thank their colleagues in the lab for the inputs and help during this work. The clinical advises from Dr V Arunkumar and Dr K V Menon are also acknowledged.

\section{References}

Baker S B, Weinzweig J, Kirschner R E and Bartlett S P 2002 Plast. Reconstr. Surg. 1091789

Bohner M 2000 Injury 31 (Suppl. 4) 37

Bohner M 2001 Eur. Spine J. 10 (Suppl. 2) S114

Cherng A M, Chow L C and Takagi S 2001 J. Endod. 27613

Comuzzi L, Ooms E and Jansen J A 2002 Clin. Oral Implants Res. 13304

Constantz B R et al 1995 Science 2671796

Csizy M, Buckley R E and Fennell C 2001 Foot Ankle Int. 22 507

Driessens F C M, Planell J A and Gil F J 1995 in Encyclopedic handbook of biomaterials and bioengineering, Part B (New York: Marcel Dekker Inc.) Vol. 2, p. 855

Driessens F C M, Planell J A, Boltong M G, Khairoun I and Ginebra M P 1998 J. Engg. Med. (Proceedings of the Institution of Mechanical Engineers, Part H) 212427

Ishikawa K, Miyamoto Y, Takechi M, Toh T, Kon M, Nagayama M and Asaoka K 1997 J. Biomed. Mater. Res. 36393

Khairoun I, Driessens F C M, Boltong M G, Planell J A and Wenz R 1999 Biomaterials 20393

Khairoun I, Magne D, Gauthier O, Bouler J M, Aguado E, Daculsi G and Weiss P 2002 J. Biomed. Mater. Res. 60 633

Komath M and Varma H K 2002 Injectable calcium phosphate cement for dental applications, Indian Patent (Applied)

Komath M, Varma H K and Sivakumar R 2000 Bull. Mater. Sci. 23135

Larsson S and Bauer T W 2002 Clin. Orthop. 39523

Lew D, Rubey T, Krizan K and Keller J C 2000 Implant Dent. 945 
Lobenhoffer P, Gerich T, Witte F and Tscherne H $2002 J$. Orthop. Trauma. 16143

Ooms E M, Wolke J G, Van Der Waerden J P and Jansen J A 2002 J. Biomed. Mater. Res. 619

Philips R W 1991 in Science of dental materials (Philadelphia: W B Saunders Co) 9th ed., p. 74

Reddi S P, Stevens M R, Kline S N and Villanueva P 1999 J. Craniomaxillofac. Trauma 57

Rehman I and Bonfield W 1995 in Bioceramics (Oxford: Elsevier Science) Vol. 8, p. 163
Sugawara A, Fujikawa K, Kusama K, Nishiyama M, Murai S, Takagi S and Chow L C 2002 J. Biomed. Mater. Res. 61 47

Sutherland I W 1991 in Biomaterials (ed.) David Byrom (NY: Stockton Press) p. 309

Turk J B and Parhiscar A 2000 Facial Plast. Surg. 167

Verheggen R and Merten H A 2001 Acta Neurochir(Wien) 143 919

Verlaan J J, van Helden W H, Oner F C, Verbout A J and Dhert W J 2002 Spine 27543 